\title{
A modified rotating isosceles triangle osteotomy using a 3D-printed patient-specific guide for the treatment of cubitus varus in children: a case report and literature review
}

\author{
Wenbing Wan ${ }^{1, \#}$, Weidong $\mathrm{Wu}^{3 \#}$, Guodong $\mathrm{Li}^{4}$, Shixin $\mathrm{Pan}^{4}$, Enmou $\mathrm{He}^{4}$, Bin $\mathrm{Hu}^{3}$, Bin $\mathrm{Shan}^{3}$, \\ Chun Chen ${ }^{2}$
}

${ }^{1}$ Department of Orthopedics, The Second Affiliated Hospital of Nanchang University, Nanchang, China; ${ }^{2}$ Department of Orthopaedic Surgery, The First Affiliated Hospital of Wenzhou Medical University, Wenzhou, China; ${ }^{3}$ State Key Laboratory of Material Processing and Die and Mould Technology and School of Materials Science and Engineering, Huazhong University of Science and Technology, Wuhan, China; ${ }^{4}$ Orthopaedic Department, Wuzhou Red Cross Hospital, Wuzhou, China

"These authors contributed equally to this work.

Correspondence to: Chun Chen, MD. Department of Orthopedics, The First Affiliated Hospital, Wenzhou Medical University, Nan Baixiang Road, Shang Caizuang Village, Wenzhou 325000, China. Email: chenchunkk@163.com; Bin Shan, PhD. School of Materials Science and Engineering, Huazhong University of Science and Technology, 1037 Luoyu Road, Wuhan 430074, China. Email: bshan@mail.hust.edu.cn.

\begin{abstract}
After corrective osteotomy of cubitus varus, the lateral condylar prominence is a common problem, which is believed to be due to the unequal relative cuts of the lateral base wedge osteotomy. Therefore, several related solutions have been proposed, such as dome osteotomy and step-cut osteotomies, which solve the above problems to a certain extent. This study aimed to: (I) use a modified corpectomy to correct the deformity, and (II) present a new corpectomy method that uses a 3D-printed specific guide with an isosceles triangle osteotomy. A 12-year-old male presented with a -30-degree cubitus varus deformity 5 years after a supracondylar fracture of the right humerus. The degree of correction was determined from the varus angle and the normal carrying angle on the normal side. A rotating isosceles triangle osteotomy was determined by using Mimics software. The accuracy of the osteotomy angle was confirmed by postoperative radiography. The mean postoperative carrying angle was found to be preserved at the 10-month followup, with no complications. A rotating isosceles triangle osteotomy with a $3 \mathrm{D}$-printed patient-specific guide may be providing a relative accurate result. However, in order to obtain more rigorous research conclusions, more cases should be added to examine this methodology for bone deformity surgery in the near future.
\end{abstract}

Keywords: Isosceles triangle osteotomy; cubitus varus; children; 3D-print patient-specific guide; case report

Submitted Mar 17, 2020. Accepted for publication Oct 26, 2020.

doi: $10.21037 / \mathrm{tp}-20-101$

View this article at: http://dx.doi.org/10.21037/tp-20-101

\section{Introduction}

Cubitus varus is a relatively common, albeit problematic, orthopedic complication resulting from the malunion of a pediatric supracondylar fracture of the humerus $(1,2)$. The elbow varus deformity could not only affects limb appearance, but also causes changes to the normal structure of the elbow joint, thereby leading to or accompanying with joint dysfunction, such as humeral pulley dysplasia, elbow joint instability, pain, weakness, and difficulty carrying weights $(1,3)$. The treatment of cubitus varus includes conservative treatment and surgery. Although the indication for surgery for cubitus varus is controversial, many previous reports tend to have an operation for relieving appearance and physical pain (1-8). Several methods of corrective osteotomy, such as step-cut osteotomy, dome osteotomy, multiplanar osteotomy, lateral wedge osteotomy, and ladder osteotomy, have been undertaken in the management of cubitus varus $(1,4)$. With conventional corrective osteotomy, 

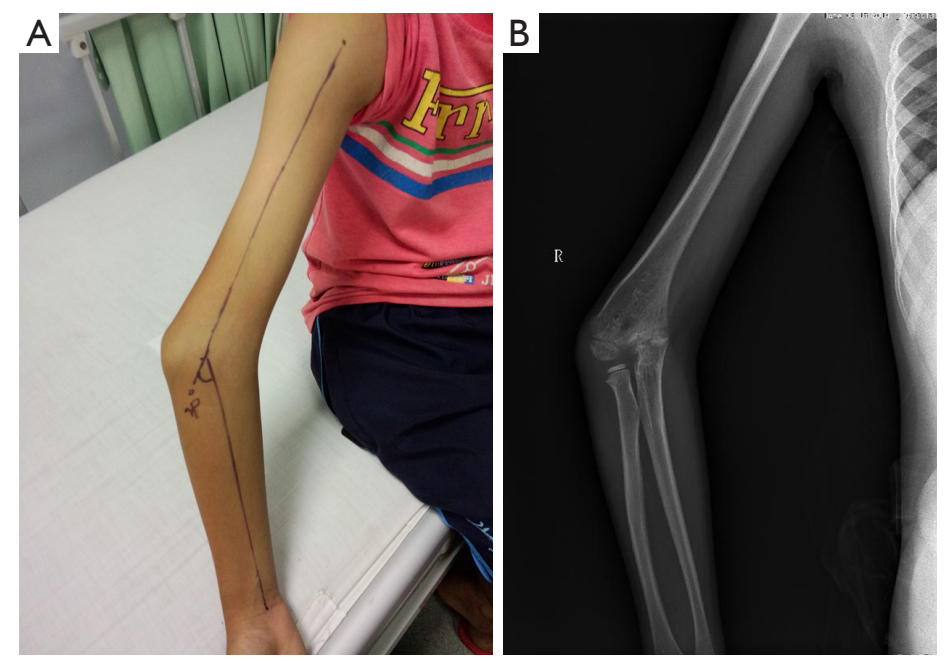

Figure 1 Preoperative photographs (A) and X-ray (B) of the patient depicting his deformity.

however, a certain degree of deformity persists because it is not always easy to correct complex $3 \mathrm{D}$ bone deformities with preoperative planning merely on the basis of plain radiographs in a free-hand operation (5). Lateral closing wedge osteotomy, the simplest of all osteotomy methods, generally results in poor bone contact and considerable blood loss as well as lateral condylar prominence that negatively affect patient expectations (6-8). Some methods, such as, dome osteotomy and step-cut osteotomies also aim to eliminate the lateral prominence $(3,9)$. However, there are still some residuals by these methods. Lately, planning using computerized models and patient-specific instrumentation have been advocated to overcome this problem (10-12).

This study aimed to present a modified surgical method that uses a $3 \mathrm{D}$-printed specific guide with an isosceles triangle osteotomy. Herein, we describe an isosceles triangle osteotomy assisted by 3D computer model planning and custom osteotomy guide method, which was very simple overall and seems to eliminate the abovementioned problems. Moreover, it provides the surgeon 3D-printed bone models that are useful for determining bone fixation options to help secure fixation; this, in return, helps the patient to start vigorous range-of-motion exercises. We present the following case in accordance with the CARE reporting checklist (available at http://dx.doi.org/10.21037/tp-20-101).

\section{Case presentation}

A 12-year-old male presented with a -30-degree cubitus varus deformity, without rotation, flexion, or extension deficits, that was the sequelae from a right humerus supracondylar fracture sustained 5 years earlier (July 21, 2014; Figure 1A,B). A target carrying angle of 10 degrees was designed; therefore, we had to cut the bone in a nearly 40-degree-angle wedge shape. Written informed consent was obtained from the patient's parents. The study was approved by ethics board of the First Affiliated Hospital of Wenzhou Medical University (No. 2014-0124) and informed consent was taken from the patient's parents. All procedures performed in studies involving human participants were in accordance with the ethical standards of the institutional and with the Helsinki Declaration (as revised in 2013).

The patient had a reasonable range of motion, but complained of mild to moderate pain during and after strenuous activities involving the affected limb. Moreover, he was very upset with the appearance of his arm. We obtained scan image data of the affected and contralateral normal elbow (including the upper arm and forearm) from our patient, who underwent $\mathrm{X}$-ray radiography and a spiral 3D computed tomography (CT) scan (LightSpeed VCT, GE, Fairfield, CT, USA) using a $0.625-\mathrm{mm}$ slice thickness and $0.30-\mathrm{mm}$ in-plane resolution. Data were transferred via a Digital Imaging and Communications in Medicine (DICOM) network into a computer workstation. Thereafter, 3D models of the affected bones were constructed by using Mimics v14.0 software (Materialise, Ltd., Belgium) and saved in the stereolithography (STL) format (Figure 2A,B,C,D).

After segmentation and reconstruction, STL data files 

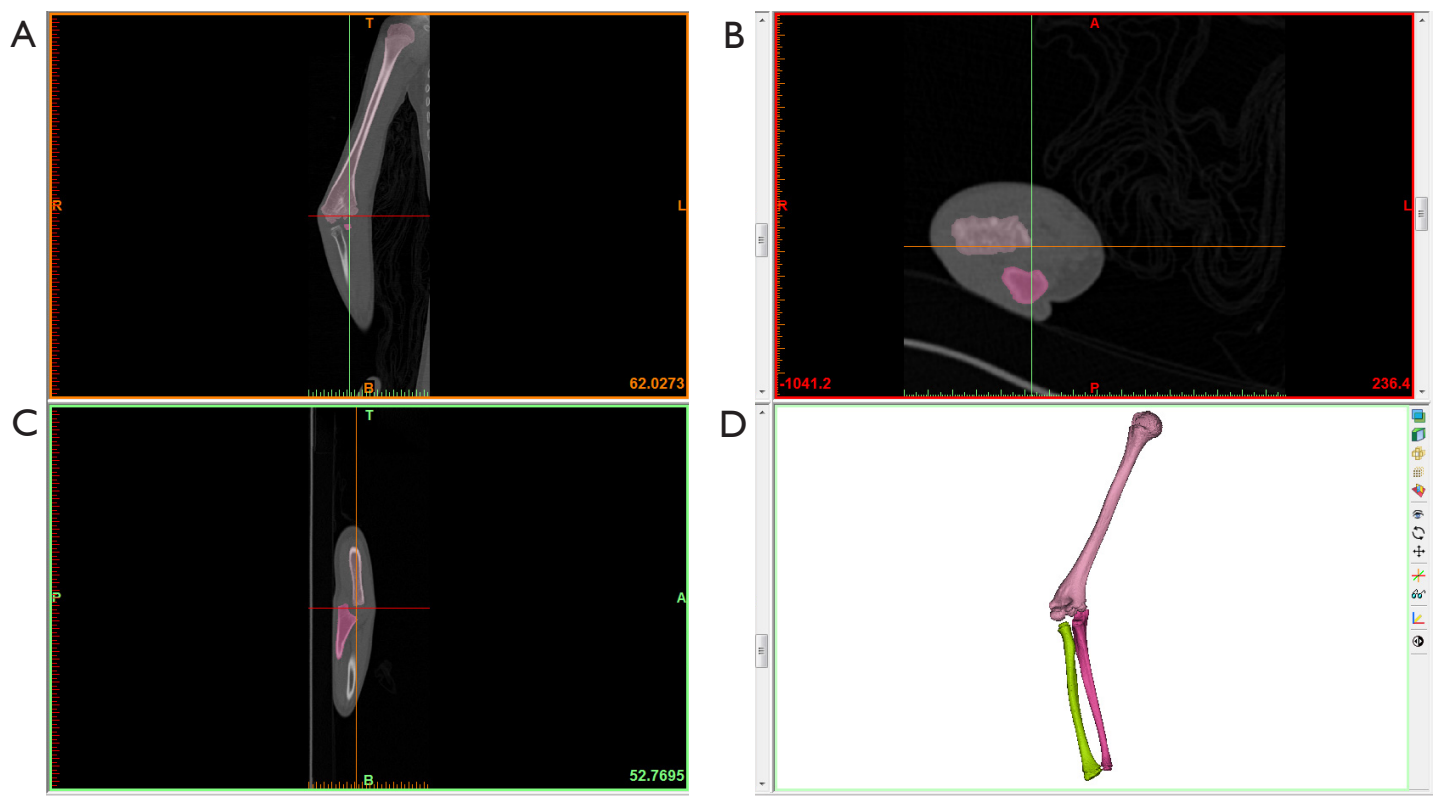

Figure 2 Three-dimensional models of the affected bones were constructed in Mimics software. (A) CT shows the coronal section of the elbow; (B) CT shows the transverse section of the elbow; (C) CT shows the sagittal section of the elbow; (D) 3D reconstruction of elbow joints. CT, computed tomography.

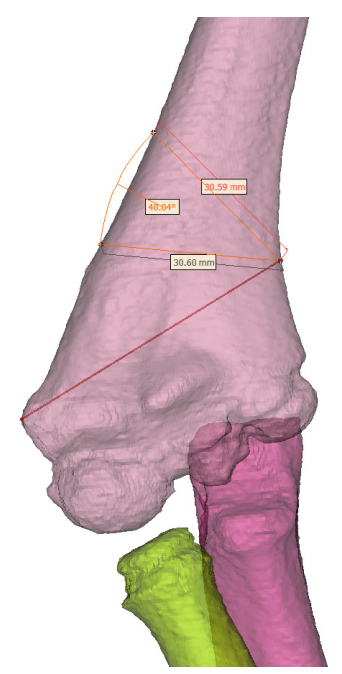

Figure 3 The isosceles triangle osteotomy was measured by Mimics software.

were imported to Geomagic Studio v2013 software (3D Systems, Ltd., USA) to correct errors and regional burrs and, then, to simulate possible osteotomy options and results. The carrying angle of the contralateral unaffected bone and exact osteotomy angle were accurately measured. The osteotomy angle was equal to the carrying angle of the contralateral unaffected bone plus the cubitus varus angle, with which one can calculate the length of the lateral base of the isosceles triangle as well as the lateral osteotomy length. The detailed procedure of the isosceles triangle osteotomy (Figure 3) was as follows: a line was drawn between the lateral epicondyle of the humerus and medial condyle of the humerus above the peak of the coronary fossa (or olecranon fossa) as one side of the isosceles triangle osteotomy; then, the medial condyle of the humerus was taken as the peak, and the other edge was drawn by a pre-calculated osteotomy angle. The triangle was rotated clockwise or anticlockwise (depending on whether the right or left upper limb was being operated upon) with the same pre-calculated angle. The rotation was stopped and the location of the osteotomy was determined when the two edges were equal.

After the osteotomy 3D simulation, the wedge-shaped bones on the anterior and posterior supracondylar aspects of the humerus were both designed as isosceles triangles. The first level of the medial hinge was closer to the joint immediately above the olecranon or coronary fossa with a 40-degree distal osteotomy cut. The range of the wedge osteotomy was defined just above the supracondylar humerus at approximately $30 \mathrm{~mm}$ (Figure $4 A$ ). We then simulated a closing wedge osteotomy for angular correction, followed by correction on the osteotomy plane based on the 
deformity evaluation (Figure 4B,C).

The distal segment of the humerus was repositioned medially on the osteotomy plane to align the lateral cortical
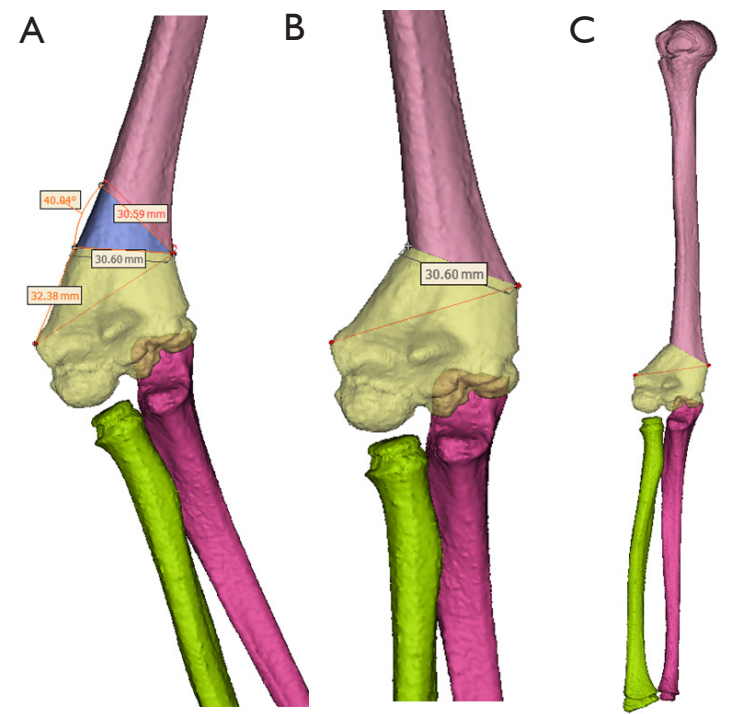

Figure 4 The simulated closed-wedge osteotomy was done in Mimics software. (A) The first level of the medial hinge was closer to the joint immediately above the olecranon or coronary fossa; (B,C) a closing wedge osteotomy for angular correction was simulated, followed by correction on the osteotomy plane based on the deformity evaluation. bone line, and there was no prominence of the lateral condyle. The proposed osteotomy was further simulated by obtaining $3 \mathrm{D}$ printouts and rehearsed with the available plate options (Figure $5 \mathrm{~A}$ ). Then an osteotomy template was designed according to the anatomical characteristics of the range of osteotomy (Figure 5B). A K-wire hole was designed for fixation in the humerus to avoid movement of the template. The osteotomy template models were exported in STL format and formed by using the Fused Deposition Modeling (FDM) rapid prototyping (RP) technique and were sterilized for standby application (Figure 5C).

\section{Results}

A posterolateral longitudinal skin incision was made along the lower arm and the full distal humerus was exposed. The osteotomy template was inserted as a "collar" to the metaphysis and diaphysis of the distal humerus at the pre-planned surface of the bone, followed by a $\mathrm{K}$-wire fixation. The bone was osteotomized with a saw along the outline of the template, and the deformity was corrected as simulated preoperatively (Figure 6A,B). After obtaining the one and only $\mathrm{C}$-arm verification to prove implementation was carried out as planned, we carried out a one-plate and four-screw fixation to complete the procedure.

The postoperative $\mathrm{X}$-ray of the patient showed the satisfying conformity of the plate and screw fixation with a
A

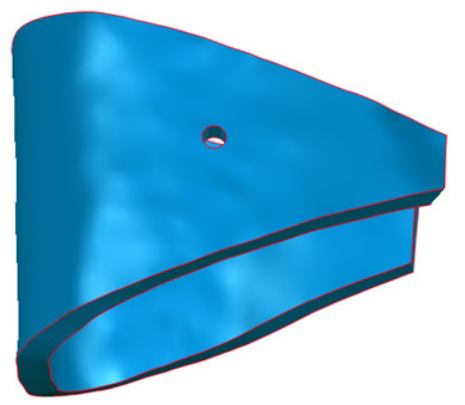

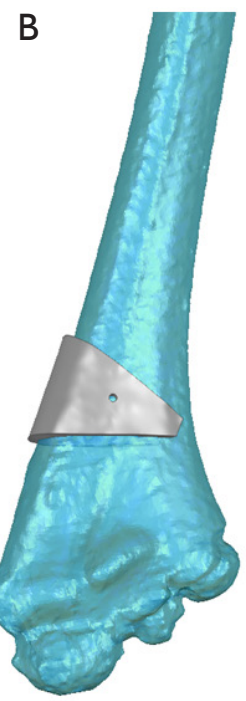

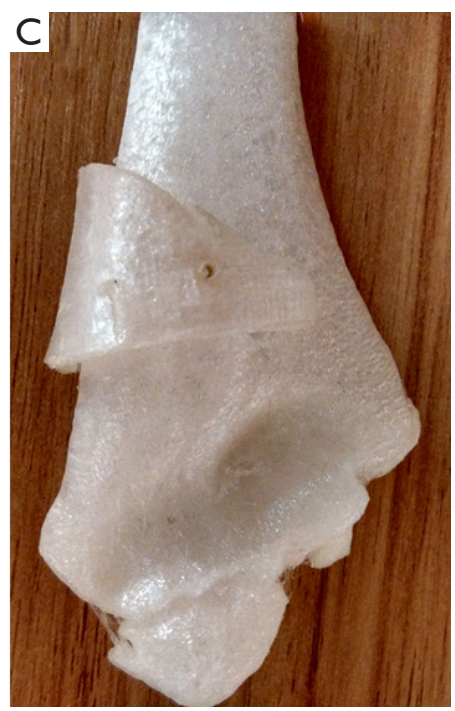

Figure 5 Graphics simulation and 3D printout rehearsals for surgery when done with our method. (A) The proposed osteotomy was further simulated by obtaining 3D printouts and rehearsed with the available plate options by software; (B) an osteotomy template was designed according to the anatomical characteristics of the range of osteotomy by software; (C) the osteotomy template models were sterilized for standby application. 

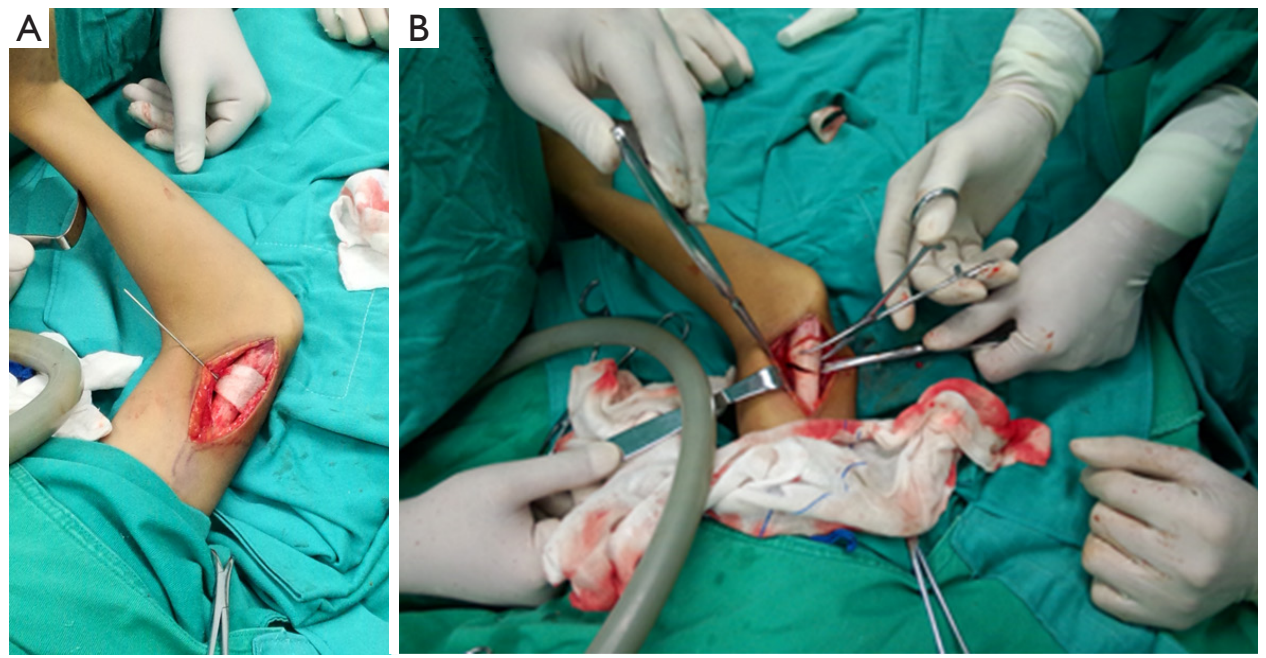

Figure 6 Photographs showing the osteotomy and resulting isosceles triangle osteotomy graft. (A) The osteotomy template was inserted as a "collar" to the metaphysis and diaphysis of the distal humerus at the pre-planned surface of the bone, followed by a K-wire fixation; (B) the bone was osteotomized with a saw along the outline of the templat.
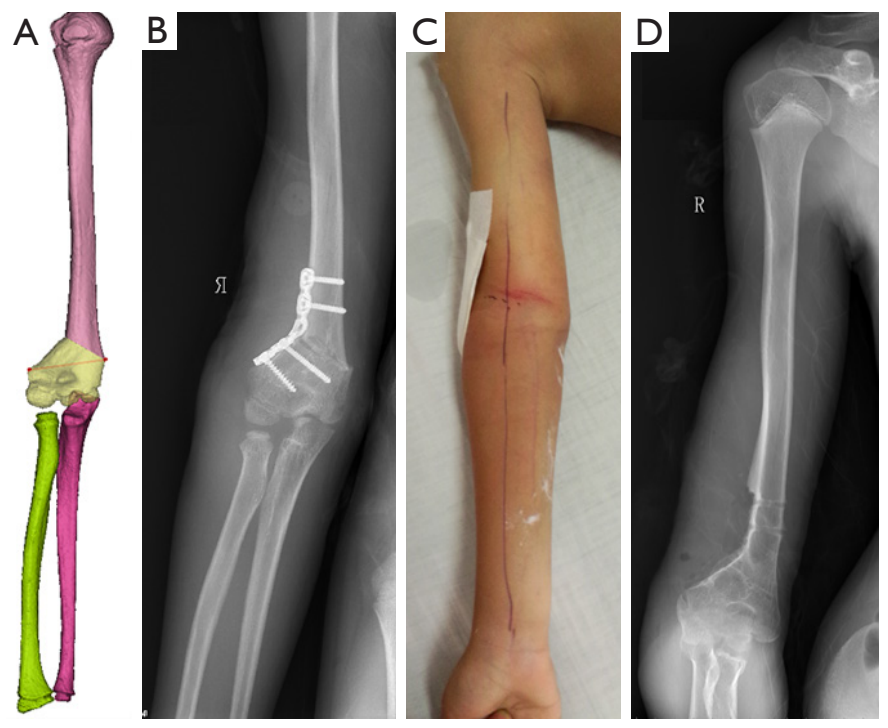

Figure 7 Early postoperative $\mathrm{X}$-rays showing the alignment, final fixation, and barely visible osteotomy lines (A,B). (C,D) show the plate removal and morphology of the upper limb.

barely visible, faint osteotomy line that satisfying matched the preoperative plan (Figure $7 A, B$ ). This indicated bone contact and fixation, and the patient was encouraged to start active and passive elbow exercises 5 days after surgery without any external splint. The patient had no pain around the joint, with 0 -degree extension and 140-degree flexion and without any lateral condylar prominence at the fourth week. X-rays revealed osteotomy site union after 3 months, and the plate and screws were removed after 10 months (Figure $7 C, D$ ). The postoperatively measured carrying angle was 9.6 degrees (against a target carrying angle of 10 degrees).

\section{Discussion}

The cubitus varus deformity is one of the most common 
complications of supracondylar fractures of the humerus, which generally include varus, internal rotation, and hyperextension deformities $(10,13,14)$. However, the etiology remains unclear. Various techniques of corrective osteotomy are available for the correction of cubitus varus (1). At present, there is no gold standard surgical correction technique for cubitus varus, and there are multiple types of osteotomies to correct these deformities in children. Firstly, conventional preoperative planning based on radiographs and manual examination affords limited accuracy $(11,12,15)$. Secondly, it is technically demanding to reproduce preoperative planning during actual surgery (16). Thirdly, lateral wedge osteotomies are conducted most commonly, and the technique is simple, effective, and reproducible. Nonetheless, multiple complicationsmost notably, the lateral condylar prominence and non$3 \mathrm{D}$ complex osseous deformities-have been documented and have compelled many surgeons to consider alternate approaches (1). Although a 3D osteotomy can correct flexion, extension, varus, valgus, and rotational deformity simultaneously (11), it cannot avoid a lateral shift and also maintain correction due to the small area of bone contact in a non-triangle osteotomy (11).

With the development of digital medicine, 3D planning and printed specific osteotomy guides are very promising options, offering shorter surgical time, flawless osteotomy, ideal fixation, and, probably, an overall lower rate of complications $(10,17,18)$. With these techniques, we developed a modified rotating triangle osteotomy for the treatment of cubitus varus in children by using a 3D-printed patient-specific guide. Many authors have reported the usefulness of 3D printing in surgery. Zhang et al. (4) treated 18 patients using a computer-aided design osteotomy template, and their results have proved highly accurate in osteotomies for correction of the cubitus varus deformity in teenagers. Omori et al. (13) treated 17 patients who underwent $3 \mathrm{D}$ corrective osteotomy by using a custommade surgical guide; the results appeared good for varusvalgus rotation, flexion-extension rotation internal-external rotation, proximal-distal translation, and carrying angle in the clinical follow-up period. We followed this guide and used a 40-degree distal osteotomy based on preoperative measurements. Based on our results, we found that an accurate osteotomy can be pre-planned by using Mimics software, and there is good bone contact after the osteotomy. Moreover, the osteoepiphysis and joint capsule are not injured in this procedure.

We modified and designed a $\mathrm{K}$-wire hole while avoiding movement of the guide, which could ensure the accuracy of the osteotomy. Moreover, some traditional osteotomies cannot avoid bone protrusion in the coronal plane. These problems can be resolved by the new rotating isosceles triangle osteotomy, based on the preoperative planning and intraoperative application. Although there a slight angle persists in the sagittal plane, new research has shown that correction of internal rotation is not needed for osteotomies that aim to correct a cubitus varus deformity (16). Usui et al. (12) measured different angles and length of osteotomy of cubitus varus, and their results have proved that a level of the medial hinge closer to the joint and immediately above the olecranon fossa is the best configuration to minimize lateral condylar prominence and the step-off of the cut surfaces, and results in a similar appearance to that of a normal humerus by using the Mimics software.

Furthermore, we did not find complications reported by previous reviews such as osteotomy angle loss, recurrence of malformation, failure of screw fixation, needle-tract infection, and a high level of osteotomy resulting in delayed bone healing and external fixation with subsequent muscular atrophy $(7,19,20)$. Moreover, the tool closing of the periosteum on the ulnar side of the distal humerus can protect the ulnar nerve during osteotomy (21). Takagi et al. (16) used an intercalary osteotomy (which flips 180 degrees in the coronal plane) technique to resolve poor bone contact and possible fixation difficulties with the use of simulating software. Our research has the following shortcomings: Firstly, we did not encounter this problem mentioned above in our modified technique. Secondly, because of the small sample size, statistical analysis was not appropriate. Thirdly, we expect more cases for verifying the safety and efficiency for the treatment of cubitus varus in children by using of the method. Moreover, relevant 3D modeling software needs to be used in the research, which puts forward higher requirements for operators, which may not be conducive to large-scale applications.

\section{Conclusions}

Our results indicate that an isosceles triangle osteotomy can generate good outcomes, and individual templates eliminate the need for complex equipment and time-consuming procedures in the operating room. This individual template can indicate the optimum pattern and plane of corrective osteotomy by calculating the axis and amount of $3 \mathrm{D}$ deformity. 


\section{Acknowledgments}

Funding: This work was supported by the Classroom Instructional Reform Research Topic of Wenzhou Medical University (YBKG201843), the National Natural Science Foundation of China (81702132), the Projects of Medical and Health Technology Program in Zhejiang Province (2017KY456) and the Wenzhou Public Welfare Science and Technology Research Project (no. Y20160130). Outstanding Young Scientist Foundation of Jiangxi Province (2018ACB21039) and Foundation of Health Commission of Jiangxi Province (20175230 and 20195228).

\section{Footnote}

Reporting Checklist: The authors have completed the CARE reporting checklist. Available at http://dx.doi.org/10.21037/ tp-20-101

Conflicts of Interest: All authors have completed the ICMJE uniform disclosure form (available at http://dx.doi. org/10.21037/tp-20-101). The authors have no conflicts of interest to declare.

Ethical Statement: The authors are accountable for all aspects of the work in ensuring that questions related to the accuracy or integrity of any part of the work are appropriately investigated and resolved. All procedures performed in studies involving human participants were in accordance with the ethical standards of the institutional and with the Helsinki Declaration (as revised in 2013). Written informed consent was obtained from the patient's parents for publication of this study and any accompanying images. Affidavit of approval of human ethics committee used protocol, the first affiliated hospital of Wenzhou medical university (2014-0124).

Open Access Statement: This is an Open Access article distributed in accordance with the Creative Commons Attribution-NonCommercial-NoDerivs 4.0 International License (CC BY-NC-ND 4.0), which permits the noncommercial replication and distribution of the article with the strict proviso that no changes or edits are made and the original work is properly cited (including links to both the formal publication through the relevant DOI and the license). See: https://creativecommons.org/licenses/by-nc-nd/4.0/.

\section{References}

1. Bauer AS, Pham B, Lattanza LL. Surgical correction of cubitus varus. J Hand Surg Am 2016;41:447-52.

2. O'Hara LJ, Barlow JW, Clarke NM. Displaced supracondylar fractures of the humerus in children. Audit changes practice. J Bone Joint Surg Br 2000;82:204-10.

3. Davids JR, Lamoreaux DC, Brooker RC, et al. Translation step-cut osteotomy for the treatment of posttraumatic cubitus varus. J Pediatr Orthop 2011;31:353-65.

4. Zhang YZ, Lu S, Chen B, et al. Application of computeraided design osteotomy template for treatment of cubitus varus deformity in teenagers: a pilot study. J Shoulder Elbow Surg 2011;20:51-6.

5. Su Y, Nan G. Lateral closing isosceles triangular osteotomy for the treatment of a post-traumatic cubitus varus deformity in children. Bone Joint J 2016;98B:1521-5.

6. Solfelt DA, Hill BW, Anderson CP, et al. Supracondylar osteotomy for the treatment of cubitus varus in children: a systematic review. Bone Joint J 2014;96-B:691-700.

7. Pannu GS, Eberson CP, Abzug J, et al. Common errors in the management of pediatric supracondylar humerus fractures and lateral condyle fractures. Instr Course Lect 2016;65:385-97.

8. Mahaisavariya B, Sithiseriprateep K, Chantarapanich N, et al. Lateral condylar prominence, post corrective osteotomy of cubitus varus: a study using three-dimensional reverse engineering technique. J Med Assoc Thai 2014;97 Suppl 9:S92-6.

9. Pankaj A, Dua A, Malhotra R, et al. Dome osteotomy for posttraumatic cubitus varus: a surgical technique to avoid lateral condylar prominence. J Pediatr Orthop 2006,26:61-6.

10. Takeyasu Y, Murase T, Miyake J, et al. Three-dimensional analysis of cubitus varus deformity after supracondylar fractures of the humerus. J Shoulder Elbow Surg 2011;20:440-8.

11. Uchida Y, Ogata K, Sugioka Y. A new three-dimensional osteotomy for cubitus varus deformity after supracondylar fracture of the humerus in children. J Pediatr Orthop 1991;11:327-31.

12. Usui M, Ishii S, Miyano S, et al. Three-dimensional corrective osteotomy for treatment of cubitus varus after supracondylar fracture of the humerus in children. J Shoulder Elbow Surg 1995;4:17-22. 
13. Omori S, Murase T, Oka K, et al. Postoperative accuracy analysis of three-dimensional corrective osteotomy for cubitus varus deformity with a custom-made surgical guide based on computer simulation. J Shoulder Elbow Surg 2015;24:242-9.

14. El-Adl W. The equal limbs lateral closing wedge osteotomy for correction of cubitus varus in children. Acta Orthop Belg 2007;73:580-7.

15. Orbach H, Rozen N, Rubin G, et al. Outcomes of French's corrective osteotomy of the humerus for cubitus varus deformity in children. Isr Med Assoc J 2018;20:442-5.

16. Takagi T, Takayama S, Nakamura T, et al. Supracondylar osteotomy of the humerus to correct cubitus varus: do both internal rotation and extension deformities need to

Cite this article as: Wan W, Wu W, Li G, Pan S, He E, Hu B, Shan B, Chen C. A modified rotating isosceles triangle osteotomy using a $3 \mathrm{D}$-printed patient-specific guide for the treatment of cubitus varus in children: a case report and literature review. Transl Pediatr 2021;10(1):215-222. doi: $10.21037 / \mathrm{tp}-20-101$ be corrected? J Bone Joint Surg Am 2010;92:1619-26.

17. Docquier PL, Paul L, TranDuy K. Surgical navigation in paediatric orthopaedics. EFORT Open Rev 2017;1:152-9.

18. Tricot M, Duy KT, Docquier PL. 3D-corrective osteotomy using surgical guides for posttraumatic distal humeral deformity. Acta Orthop Belg 2012;78:538-42.

19. Raney EM, Thielen Z, Gregory S, et al. Complications of supracondylar osteotomies for cubitus varus. J Pediatr Orthop 2012;32:232-40.

20. Bouton D, Ho CA, Abzug J, et al. The difficult supracondylar humerus fracture: flexion-type injuries. Instr Course Lect 2016;65:371-7.

21. Mitsunari A, Muneshige H, Ikuta Y, et al. Internal rotation deformity and tardy ulnar nerve palsy after supracondylar humeral fracture. J Shoulder Elbow Surg 1995;4:23-9. 